\title{
Varied Presentation and Importance of MR Neurography of the Common Fibular Nerve in Slimmer's Paralysis
}

\author{
Lisa B.E. Shields ${ }^{a} \quad$ Vasudeva G. Iyer ${ }^{b}$ Christopher B. Shields ${ }^{a, c}$ \\ Yi Ping Zhang ${ }^{\text {a }}$ Abigail J. Rao ${ }^{a}$ \\ aNorton Neuroscience Institute, Norton Healthcare, Louisville, KY, USA; beurodiagnostic \\ Center of Louisville, Louisville, KY, USA; 'Department of Neurological Surgery, University of \\ Louisville School of Medicine, Louisville, KY, USA
}

\section{Keywords}

Neurology · Slimmer's paralysis · Common fibular nerve · Magnetic resonance neurography · Electromyography

\begin{abstract}
Slimmer's paralysis refers to a common fibular nerve palsy caused by significant and rapid weight loss. This condition usually results from entrapment of the common fibular nerve due to loss of the fat pad surrounding the fibular head. Several etiologies of common fibular nerve palsy have been proposed, including trauma, surgical complications, improperly fitted casts or braces, tumors and cysts, metabolic syndromes, and positional factors. We present 5 cases of slimmer's paralysis in patients who had lost $32-57 \mathrm{~kg}$ in approximately 1 year. In 2 cases, MR neurogram of the knee demonstrated abnormalities of the common fibular nerve at the fibular head. Two patients underwent a common fibular nerve decompression at the fibular head and attained improved gait and sensorimotor function. Weight loss, diabetes mellitus, and immobilization may have contributed to slimmer's paralysis in 1 case. Awareness of slimmer's paralysis in patients who have lost a significant amount of weight in a short period of time is imperative to detect and treat a fibular nerve neuropathy that may ensue.
\end{abstract}

\section{Introduction}

Woltman [1] reported and Sprofkin [2] subsequently corroborated a correlation between weight loss and crossing the legs with the development of fibular nerve palsy [1,2]. Initially coined by Sotaniemi [3] in 1984, slimmer's paralysis denotes fibular nerve paralysis that 
develops after substantial weight reduction. The proposed mechanism is loss of adipose tissue surrounding the nerve resulting in entrapment of the common fibular nerve at the fibular head, with subsequent ischemia and edema of the common fibular nerve and impaired axonal transport [3-6]. The pathophysiology involves focal demyelination with axonal involvement at the fibular head or axonal degeneration without involvement of the covering myelin [7]. Symptoms include motor and sensory deficits in the distribution of the common fibular nerve, including weakened foot eversion and dorsiflexion, toe dorsiflexion, and gait abnormalities.

Slimmer's paralysis may be due to a mechanical compressive neuropathy, metabolic alterations, microcirculatory disturbances, or a combination of all 3 [3-6, 8-10]. It has also been suggested that inflammation and altered immunity may play a role in the pathogenesis [11]. Myriad etiologies of common fibular nerve palsy include traumatic injury to the fibular head, surgical complications, improperly fitted casts or braces, prolonged bedridden patients, tumor, cyst, leg crossing, and paraneoplastic and metabolic syndromes $[5,8,9]$. Due to the lack of studies on the differential diagnosis of slimmer's paralysis, the aim of the current case series was to investigate the differential diagnosis of this disorder using the techniques of MR neurography combined with EMG/NCV studies and/or ultrasound (US).

Herein, we present 5 cases of slimmer's paralysis resulting from significant weight loss during a brief time period. We discuss the differential diagnosis of this condition and the electrodiagnostic and imaging studies that confirm it. The nerve conduction studies were performed as per published American Association of Neuromuscular \& Electrodiagnostic Medicine guidelines [12]. When compound muscle action potentials (CMAP) were absent or too small to measure the latency and amplitude accurately, the nerve conduction studies were done with the recording electrode over the tibialis anterior and peroneus longus (PL) with stimulation at the fibular neck and the popliteal fossa.

\section{Case Descriptions}

\section{Case 1}

History, Physical Examination, and Radiological Imaging

A 65-year-old woman presented with numbness and weakness in the feet that radiated proximally to the mid-calves (Table 1). She reported a 2-week history of spontaneous left foot drop. She also reported stumbling gait and toe spasms. The patient had lost $41 \mathrm{~kg}$ in the past year without bariatric surgery. Medical history was significant for optic neuritis. Multiple sclerosis was initially considered as the cause of her symptoms but not confirmed by MRI studies. She was not known to be diabetic.

On sensory exam, pinprick sensation was decreased over the lateral aspect of the left leg. There was weakness of dorsiflexion of the left ankle and the left toes. A lumbar MRI demonstrated spinal and foraminal stenosis at L4-5. A left knee MRI with and without gadolinium contrast and MR neurogram revealed an abnormal T2 hyperintensity of the common fibular nerve and its proximal superficial and deep branches at the level of the left fibular head and fibular tunnel (Fig. 1a, b). Severe tricompartmental osteoarthritis of the left knee was observed. There was no evidence of a compressive/impinging mass, fluid collection, or ganglion cyst.

EMG/NCV of the Legs

The left common fibular nerve showed decreased motor conduction velocity across the knee. There was also a drop in the amplitude of CMAP of the extensor digitorum brevis (EDB) between stimulation at the ankle and at the fibular neck. The sensory nerve action potentials (SNAP) could not be recorded over the superficial fibular nerve. Needle EMG

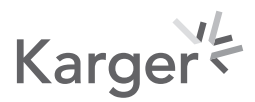



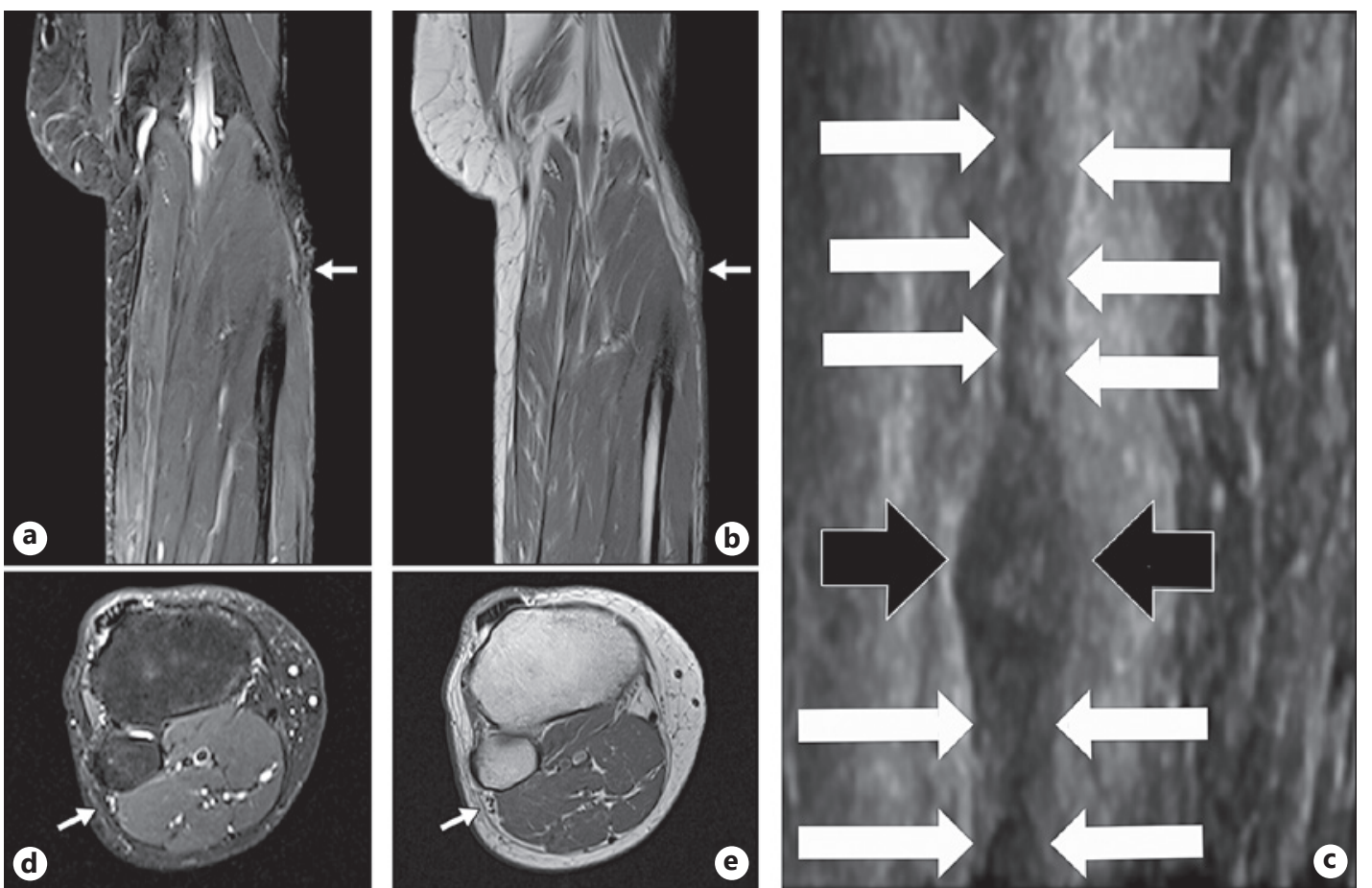

Fig. 1. In case 1, coronal STIR (a) and T1 sequences (b) show focal T2 hyperintensity and enlargement of the left common fibular nerve as the nerve crosses the fibular head (white arrows), corresponding with the ultrasound (c) at the time of the electrodiagnostic study (black arrows show the area of enlargement; white arrows point to the nerve proximal and distal to the abnormal area). In case 3, axial STIR (d) and T1 sequences (e) show T2 hyperintensity and compression of the right common fibular nerve as it crosses the fibular head at the lateral knee (white arrows).

showed decreased motor units and increased polyphasics in the left tibialis anterior (TA) and extensor hallucis longus (EHL). There was no evidence of denervation/reinnervation in the lumbar paraspinals. US demonstrated an enlarged common fibular nerve at the fibular neck (Fig. 1c). The findings confirmed left common fibular nerve neuropathy at the fibular neck.

\section{Surgical Intervention}

The patient underwent a left common fibular nerve decompression at the fibular neck. The superficial fascia and the anterior and posterior crural intermuscular septa were all grossly compressive. Within 2 weeks of this surgery, she attained excellent improvement of her sensorimotor deficits with resolution of her stumbling gait.

\section{Case 2}

History, Physical Examination, and Radiological Imaging

A 31-year-old man experienced a near drowning episode 10 months earlier. Two months later, he was diagnosed with a right lung abscess and diabetic ketoacidosis. The patient was ventilated and sedated for 4 days after which he immediately developed a bilateral foot drop (Table 1). He remained hospitalized for 26 days at which time he lost $45 \mathrm{~kg}$.

On sensory exam, decreased pinprick sensation was noted up to the mid-calves bilaterally in a stocking distribution. There was marked weakness of dorsiflexion of the ankles and of toe extension bilaterally. A lumbar MRI demonstrated no abnormal findings. 
EMG/NCV of the Legs

CMAP could not be recorded over the EDB on stimulation of the fibular nerves. The tibial nerves showed prolonged distal motor latency and decreased motor conduction velocity. No sensory potentials could be recorded on stimulation of plantar or superficial fibular nerves, and the sural potential showed a low amplitude. Needle EMG revealed denervation/reinnervation changes in the TA and EHL bilaterally with a normal pattern in the short head of the biceps femoris and the gastrocnemius muscles. The proximal EMG (hamstrings) was normal. The EMG pattern did not suggest a myopathic process. The topography of denervation/reinnervation suggested a peroneal nerve neuropathy. It was unlikely to be a critical care neuropathy.

The EMG/NCV confirmed bilateral common fibular nerve neuropathy at the fibular neck. The changes in conduction of the tibial nerves and most of the sensory nerves in the lower extremities were likely due to diabetic polyneuropathy.

Surgical Intervention

A fibular nerve decompression was performed on the right followed 2 weeks later on the left. Within 1 month of these procedures, the patient had improved gait, dorsiflexion of the left ankle, and sensation of the lower right lateral calf.

\section{Case 3}

History, Physical Examination, and Radiological Imaging

A 43-year-old woman with current tobacco abuse sustained a fall from a chair 10 days earlier following which she complained of low back pain. Three days later, she experienced right foot weakness, with an inability to pick up her foot or wiggle her toes (Table 1). She reported numbness of the right lateral leg. The patient intentionally lost $32 \mathrm{~kg}$ by dieting over the previous year. She did not have diabetes mellitus.

On sensory exam, decreased pinprick sensation over the dorsum of foot and dyesthesia over the lateral aspect of the right leg were observed. There was marked weakness of the TA, EHL, and peroneous longus. A lumbar MRI revealed a disc herniation and degenerative disc disease at L4-5 and L5-S1. A right knee MRI with and without gadolinium contrast and MR neurogram demonstrated increased signal of the common fibular nerve at the level of the fibular head and neck as it passed through the fibular tunnel (Fig. 1d, e). Tricompartmental arthropathy was detected which was severe in the patellofemoral compartment and moderate in the medial femorotibial compartment.

EMG/NCV of the Right Lower Extremity

Stimulation at the right fibular neck evoked a small CMAP over the EDB. Stimulation of the right fibular nerve evoked CMAP with a low normal amplitude over the peroneous longus; motor conduction velocity was normal across the popliteal fossa. The superficial peroneal SNAP latency was $2.9 \mathrm{~ms}$ with an amplitude of $16.1 \mathrm{uV}$. Needle EMG showed positive waves in the right TA, and motor units were not able to be recruited in the TA or EHL. US demonstrated an enlarged fibular nerve at the fibular neck. A fibular nerve neuropathy at the fibular neck was confirmed involving the deep fibular more than the superficial fibular fascicles. There were denervation/reinnervation changes and a partial conduction block.

Neurological Follow-Up

Following a 1-week course of methylprednisolone, the patient had improved motor strength on exam. She did not require surgical intervention.

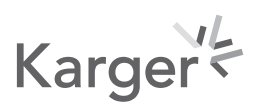




\section{Case 4}

History and Physical Examination

A 51-year-old woman presented with weakness and numbness of the right lower extremity (Table 1). She also reported "slapping" of her right foot and low back pain without radiculopathy. The patient had lost $32 \mathrm{~kg}$ of weight in the previous year through diet and exercise. Past medical history was negative for diabetes mellitus.

On sensory exam, decreased pinprick sensation was noted over the lateral aspect of the right leg and dorsum of the foot. There was also marked weakness of dorsiflexion of the right ankle and significant weakness of the everters.

EMG/NCV of the Right Leg

Stimulation of the right fibular nerve at the ankle and fibular neck evoked CMAP over the EDB with a mildly decreased amplitude. Stimulation at the popliteal fossa evoked CMAP with a significantly lower amplitude over the EDB with dispersion of the CMAP. The motor conduction velocity across the popliteal fossa was markedly decreased. Needle EMG showed decreased motor unit recruitment and increased polyphasics in the TA and EHL. The peroneous longus showed fibrillations, positive waves, and significantly decreased motor unit recruitment with an increase in polyphasics.

A right common fibular nerve neuropathy at the level of the fibular head was confirmed. The findings suggested a combination of partial conduction block, focal demyelination, and axonal involvement especially of the fascicles innervating the peroneus longus muscle.

Neurological Follow-Up

The patient's fibular nerve palsy resolved spontaneously, and, therefore, she did not require surgical intervention.

Case 5

History and Physical Examination

A 71-year-old female reported a 3-week history of right foot drop without pain or paresthesia (Table 1). She had lost $57 \mathrm{~kg}$ over several months and described that she frequently crosses her legs. Medical history was significant for diabetes mellitus. On exam, there was marked weakness of dorsiflexion of the right ankle and extension of the right toes.

EMG/NCV of the Right Leg

Stimulation of the right common fibular nerve at the ankle evoked CMAP with a low amplitude over the EDB (Fig. 2a). Stimulation at the fibular neck evoked CMAP with a much lower amplitude. No measurable potential could be recorded on stimulation in the proximal popliteal fossa. The motor nerve conduction velocity in the leg segment was decreased. Needle EMG showed positive sharp waves and fibrillations in the tibialis anterior and extensor hallucis longus. Decreased motor unit recruitment was noted in the PL. The sensory potential over the superficial fibular nerve at the ankle on antidromic stimulation was $10 \mathrm{~cm}$ proximally (Fig. 2b). The latency and amplitude were within normal limits.

A right common fibular nerve neuropathy involving the deep fibular branch at the fibular neck/fibular tunnel was confirmed. A combination of a conduction bock and axonal involvement explained the needle EMG findings.

Neurological Follow-Up

The patient was treated with an ankle and foot orthoses brace and physical therapy. She did not require surgical intervention.

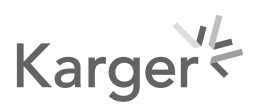



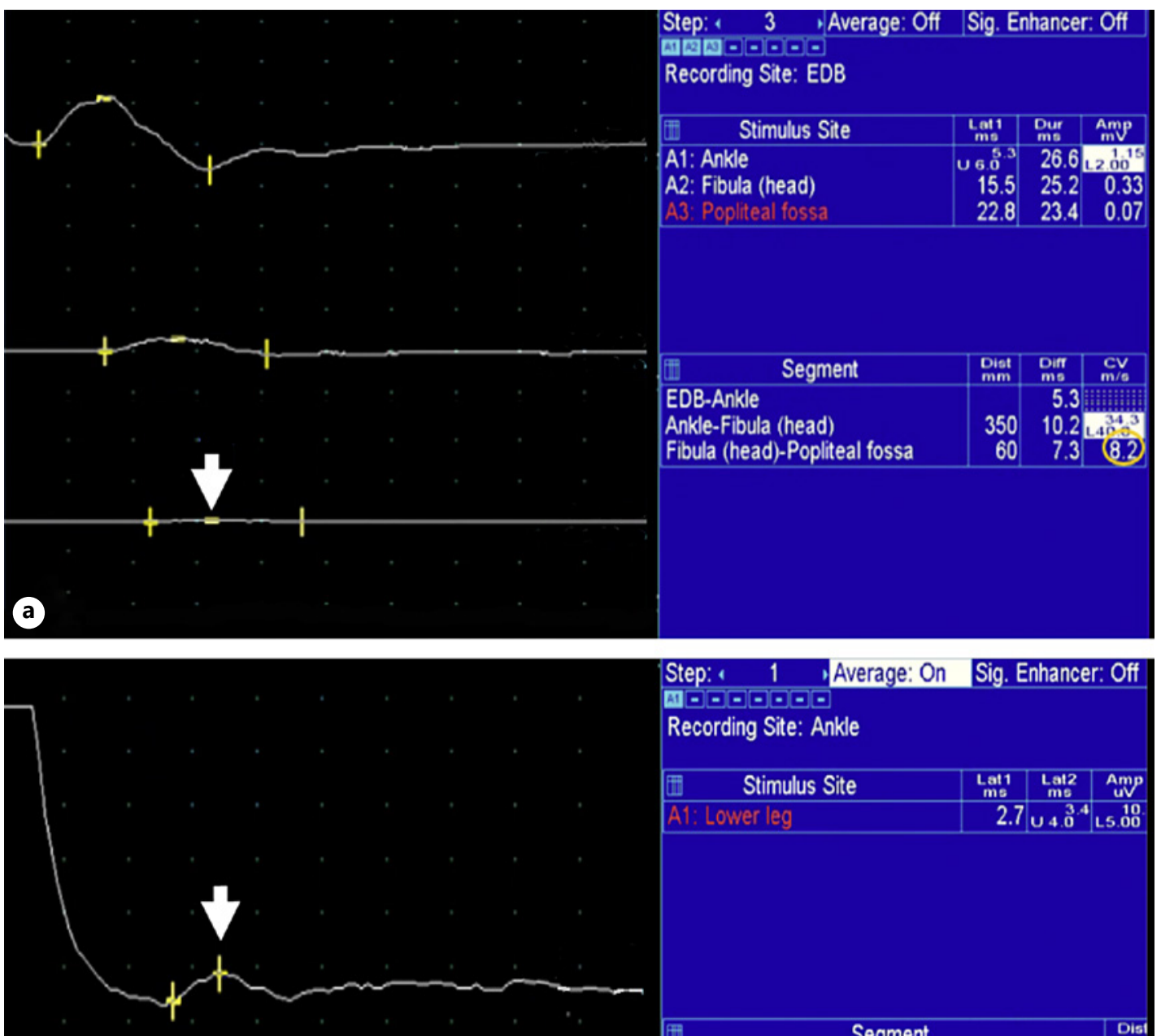

b

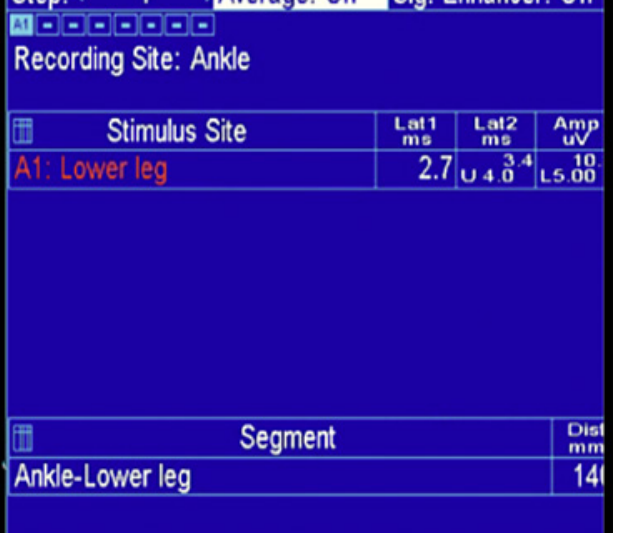

Fig. 2. a In case 5, right common fibular nerve stimulated at the ankle, fibular neck, and popliteal fossa with the recording electrode over the extensor digitorum brevis. Note the marked drop in amplitude of the compound muscle action potential on stimulation at the popliteal fossa (arrow) and significant slowing of motor conduction. The findings suggest focal demyelination with conduction block in the common fibular nerve across the fibular head. b Sensory potential over the superficial fibular nerve at the ankle (arrowhead) on antidromic stimulation was $10 \mathrm{~cm}$ proximally. The latency and amplitude were within normal limits.

\section{Discussion}

Slimmer's paralysis is marked by significant weight loss in a short period of time that results in a fibular nerve neuropathy. In the study by Katirji and Wilbourn [7] including 103 patients with common fibular nerve mononeuropathy, the majority (37 [35.9\%]) of patients developed this condition following weight loss ( $>9 \mathrm{~kg}$ over 2-3 months). Slimmer's paralysis has been described through dieting $[5,8]$ and bariatric surgery $[4,6,11,13]$. It has been reported that rapid reduction of body weight is correlated with a higher risk of developing foot drop [13]. Of note, $>1$ factor may contribute to slimmer's paralysis in a single patient such as weight reduction, inactivity, and habitual sitting with crossed legs $[3,9]$. Diabetes mellitus and cigarette smoking may also play a role in slimmer's paralysis, as demonstrated in our case series. 
Table 2. Anatomy of fibular nerve predisposes to injury leading to neuropathy

Minimal epineural supporting tissue

Tunnel may be formed between biceps femoris muscle and lateral gastrocnemius muscle; this tunnel results in less fatty cushioning of fibular nerve

More proximal bifurcation of fibular nerve

Superficial course of fibular nerve around fibular neck

Fibular tunnel is formed between musculo-aponeurotic arch of peroneus longus and soleus tendon and bony floor of proximal fibula; entrapment or impingement of this tunnel occurs between the fibers of the peroneus longus and fibular neck

Additional branches of fibular nerve at knee joint

The common fibular nerve is the lateral division of the sciatic nerve that courses from the posterolateral side of the knee around the biceps femoris tendon and the fibular head to the anterolateral aspect of the lower leg [14]. The fibular nerve has several anatomic findings that may predispose it to injury resulting in neuropathy (Table 2). The mechanism associated with slimmer's paralysis is likely due to loss of general body weight leading to fat loss around the nerves which serves to pad them.

A comprehensive history and physical examination are warranted in the investigation of slimmer's paralysis. The differential diagnosis includes sensory-predominant polyneuropathy, sciatic mononeuropathy, lumbosacral plexopathy, radiculoplexus neuropathy, L5 radiculopathy, and a fibular lesion $[7,11]$. Due to the overlap in sensorimotor findings between a lumbar radiculopathy and a fibular nerve lesion, spinal imaging is helpful to evaluate lumbar spine pathology. Electrodiagnostic studies are valuable to confirm a fibular nerve neuropathy and often depict characteristic findings such as severe slowing of conduction velocity in the above-to-below the fibular head segment, a significant reduction of CMAP, in TA, and EDB between stimulation at the popliteal fossa compared to stimulation below the fibular head, and a conduction block at the fibular head $[7,9,15]$. Furthermore, the EMG/NCV describes whether the neuropathic process is axonal, demyelinating, or a combination of both [4]. An US of the fibular nerve may also aid in the diagnosis of fibular neuropathy [10]. The primary features on US are dark spots in the fibular nerve on the pathological side which may be due to a less protective adipose tissue and intraneural edema in the fibular nerve. Three-dimensional MR neurography with its high resolution, high field, and superior T2 contrast has been shown to provide excellent visualization of the common fibular nerve [16]. Additional advances include improvements in coil technology, absence of pulsation artifacts, and isotropic acquisition with multiplanar capabilities using 3T MR scanners.

Slimmer's paralysis may be treated conservatively through a balanced diet with adequate nutritional support and vitamin supplementation [6]. A decompression of the common fibular nerve at the fibular head may be indicated when sensorimotor deficits have a sudden onset, are severe, or are persistent. Surgical decompression is recommended when symptoms persist or recovery remains incomplete after 3-4 months [15].

Our case series reflects the varied presentations of slimmer's paralysis and the importance of MR neurography in distinctly evaluating the pathology of the common fibular nerve. Additionally, the literature primarily highlights patients who developed slimmer's paralysis after bariatric surgery, while none of the 5 patients in our series underwent this procedure. While 4 patients lost a substantial amount of weight through dieting over a year, 1 patient displayed a combination of factors that may have contributed to his slimmer's paralysis including uncontrolled and undiagnosed diabetes mellitus, immobilization, and significant weight loss in 1 month. One patient frequently crossed her legs which she had been unable to

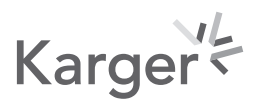


do prior to her drastic weight loss. An important aspect of our case series is that MR neurograms have not been extensively reported in slimmer's paralysis. MR neurograms in 2 patients in our series demonstrated hyperintensity of the common fibular nerve at the fibular head, and the US in 1 patient showed an enlarged fibular nerve at the fibular neck. The EMG/NCV testing on all 5 patients in our series was valuable in detecting peroneal neuropathy. The EMG/NCV findings of our 5 patients closely reflect the published literature of a fibular neuropathy $[7,9,15]$. Two patients underwent decompression of the common fibular nerve and achieved improvement of their sensorimotor function postoperatively, while 3 patients recovered through conservative measures.

The limitation of the current study is that MR neurography requires access to specialized protocols and high-resolution scanners. MR neurography needs a 3T scanner, although $1.5 \mathrm{~T}$ is more common. Furthermore, minimal patient motion is mandatory during MR neurography.

\section{Conclusion}

Due to the lack of studies on the differential diagnosis of slimmer's paralysis, this case series explored the differential diagnosis of this disorder using the techniques of MR neurography combined with EMG/NCV studies and/or US. A neuropathy at the fibular head should be considered in patients developing neurological symptoms of rapid onset following significant weight loss through bariatric surgery or dieting. A detailed history and physical examination with EMG/NCV and MR neurography are helpful to confirm the diagnosis of slimmer's paralysis. Early detection and timely treatment through either conservative measures or surgical intervention may mitigate the permanent sequelae that may develop.

\section{Acknowledgment}

We acknowledge Norton Healthcare for their continued support.

\section{Statement of Ethics}

Written informed consent was obtained from the patients for publication of this case report and any accompanying images. The University of Louisville Institutional Review Board has determined that our project does not meet the "Common Rule" definition of human subjects' research and does not require IRB review. The Institutional Review Board number is 19.1259 .

\section{Conflict of Interest Statement}

The authors have no conflicts of interest to declare.

\section{Funding Sources}

The authors declare that no funding sources were required for this case series.

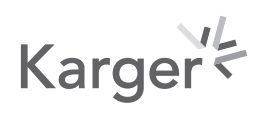




\section{Author Contributions}

Lisa B.E. Shields was involved in data conception, design, acquisition, analysis, and interpretation; drafted the manuscript; critically revised the manuscript; and gave final approval. Vasudeva G. Iyer was involved in data conception, design, acquisition, analysis, and interpretation; critically revised the manuscript; and gave final approval. Christopher B. Shields was involved in data conception, design, acquisition, analysis, and interpretation; critically revised the manuscript; and gave final approval. Yi Ping Zhang was involved in data conception, design, acquisition, analysis, and interpretation; critically revised the manuscript; and gave final approval. Abigail J. Rao was involved in data conception, design, acquisition, analysis, and interpretation; critically revised the manuscript; and gave final approval.

\section{Data Availability Statement}

All data generated or analyzed during this study are included in this article. Further enquiries can be directed to the corresponding author.

\section{References}

1 Sprofkin BE. Peroneal paralysis; a hazard of weight reduction. AMA Arch Intern Med. 1958;102:82-7.

2 Woltman HW. Crossing the legs as a factor in the production of peroneal palsy. JAMA. 1929;93(9):670-2.

3 Sotaniemi KA. Slimmer's paralysis-peroneal neuropathy during weight reduction. J Neurol Neurosurg Psychiatry. 1984;47:564-6.

4 Elias WJ, Pouratian N, Oskouian RJ, Schirmer B, Burns T. Peroneal neuropathy following successful bariatric surgery. Case report and review of the literature. J Neurosurg. 2006;105:631-5.

5 Margulis M, Ben Zvi L, Bernfeld B. Bilateral common peroneal nerve entrapment after excessive weight loss: case report and review of the literature. J Foot Ankle Surg. 2018 May;57(3):632-4.

6 Ramos-Levi AM, Matias-Guiu JA, Guerrero A, Sanchez-Pernaute A, Rubio MA. Peroneal palsy after bariatric surgery; is nerve decompresion always necessary? Nutr Hosp. 2013;28:1330-2.

7 Katirji MB, Wilbourn AJ. Common peroneal mononeuropathy: a clinical and electrophysiologic study of 116 lesions. Neurology. 1988;38:1723-8.

8 Cruz-Martinez A, Arpa J, Palau F. Peroneal neuropathy after weight loss. J Peripher Nerv Syst. 2000;5:101-5.

9 Cruz Martinez A. Slimmer's paralysis: electrophysiological evidence of compressive lesion. Eur Neurol. 1987; 26:189-92.

10 Meylaerts L, Cardinaels E, Vandevenne J, Velghe B, Gelin G, Vanormelingen L, et al. Peroneal neuropathy after weight loss: a high-resolution ultrasonographic characterization of the common peroneal nerve. Skeletal Radiol. 2011;40:1557-62.

11 Thaisetthawatkul P, Collazo-Clavell ML, Sarr MG, Norell JE, Dyck PJ. A controlled study of peripheral neuropathy after bariatric surgery. Neurology. 2004;63:1462-70.

12 Chen S, Andary M, Buschbacher R, Del Toro D, Smith B, So Y, et al. Electrodiagnostic reference values for upper and lower limb nerve conduction studies in adult populations. Muscle Nerve. 2016;54:371-7.

13 Weyns FJ, Beckers F, Vanormelingen L, Vandersteen M, Niville E. Foot drop as a complication of weight loss after bariatric surgery: is it preventable? Obes Surg. 2007;17:1209-12.

14 Van den Bergh FR, Vanhoenacker FM, De Smet E, Huysse W, Verstraete KL. Peroneal nerve: normal anatomy and pathologic findings on routine MRI of the knee. Insights Imaging. 2013;4:287-99.

15 Fabre T, Piton C, Andre D, Lasseur E, Durandeau A. Peroneal nerve entrapment. J Bone Joint Surg Am. 1998; 80:47-53.

16 Chhabra A, neurography MR. MR neurography. Neuroimaging Clin N Am. 2014;24(1):xvii. 